\title{
CORRECTION OF IONOSPHERIC DISTORTIONS IN LOW FREQUENCY INTERFEROMETRIC SAR DATA
}

\author{
Jun Su Kim, Andreas Danklmayer and Konstantinos Papathanassiou \\ German Aerospace Center (DLR) \\ Microwaves and Radar institute \\ P.O.BOX 1116, D-82230 Wessling, Germany
}

\begin{abstract}
In this paper we propose some ionospheric correction schemes for space-borne synthetic aperture radar (SAR) and polarimetric interferometric SAR (PolInSAR). The spatial and temporal variation of the free electron density in the uppermost atmosphere affects the propagation of the radar pulse resulting in image distortions. We estimate the total electron content (TEC) by applying the Appleton-Hartree equation to the distortions in the focusing, polarimetry, and interferometry. Then we propose a combined estimator that yields comprehensive differential TEC estimations. The effect of vertical structures of the ionosphere on interferometric phase is further discussed.
\end{abstract}

Index Terms - PolInSAR, ionosphere

\section{INTRODUCTION}

One of the prominent objectives of the next generation space remote sensing missions is the accurate and continuous monitoring of the terrestrial biomass distribution by means of low frequency SAR. Low frequency microwaves are characterized by higher penetration ability through vegetation foliage, as well as higher temporal stability facilitating vegetation monitoring by means of SAR and InSAR. However, the distortion due to the ionosphere is no longer negligible [1]. In this sense the estimation and compensation of the ionospheric impact is critical for the success of such missions.

According to $[1,2,3]$, the spatial and temporal distribution of TEC is the key parameter that characterizes the ionospheric impact on SAR and InSAR acquisitions. At the same time, system parameters, such as the carrier frequency $f_{0}$, the system bandwidth $W$, the incidence angle $\theta$, and the polarization are also confining factors.

In this paper we use and compare three different TEC estimators, based on ionospheric induced distortions of SAR, PolSAR and InSAR acquisitions. Each distortion is formularized as a function of TEC and system parameters. The correction scheme, the quality metric and the correction result follow. Finally, we suggest a combined scheme of TEC estimation and correction. In the discussion, we suggest that the ionospheric altitude has to be considered in the InSAR application.

\section{IONOSPHERIC IMPACT ON SAR}

\subsection{Appleton-Hartree equation}

The ionospheric impact on SAR signal and imagery can be derived starting from the Appleton-Hartree equation $[2,4,5]$. The Appleton-Hartree equation can be approximated for the space-borne SAR condition as follow:

$$
n=1-\frac{1}{2} \frac{f_{p}^{2}}{f^{2}}\left(1 \mp \frac{f_{H}}{f}\right),
$$

where $f_{p}^{2}=N e^{2} / 4 \pi^{2} \epsilon_{0} m$ is the plasma frequency and $f_{H}=$ $e \vec{B} \cdot \hat{\kappa} / 2 \pi m$ is the cyclotron frequency. The Appleton-Hartree equation provides the index of refraction $n$ of the ionosphere for a given number density of free electrons $N$ and the Earth's magnetic field $\vec{B}$, where $e$ and $m$ are the charge and mass of an electron, and $\epsilon_{0}$ is the vacuum permittivity.

\subsection{Differential Faraday rotation}

Faraday rotation (FR) owes to the anisotropic nature of the ionosphere posed in an external, or in our case, Earth's, magnetic field. The difference of two indices of refraction is from Eq. (1)

$$
\Delta n=\frac{N \zeta e \vec{B} \cdot \hat{\kappa}}{\pi m f_{0}^{3}}
$$

where

$$
\zeta=\frac{e^{2}}{8 \pi^{2} \epsilon_{0} m}=\frac{e^{2} c^{2}}{2 \pi m} \times 10^{-7}=40.3082 \mathrm{~m}^{3} / \mathrm{s}^{2} .
$$

Integrating Eq. (2) along the line-of-sight (LOS) yields the phase difference between two characterizing waves

$$
2 \Omega=\Delta \phi=2 \pi f_{0} t=\frac{2 \pi f_{0}}{c} \int_{0}^{S} \Delta n \mathrm{~d} l .
$$

Here $\Omega$ stands for the FR angle, $\mathrm{d} l$ is the infinitesimal length element, and the integration interval goes from the target to 


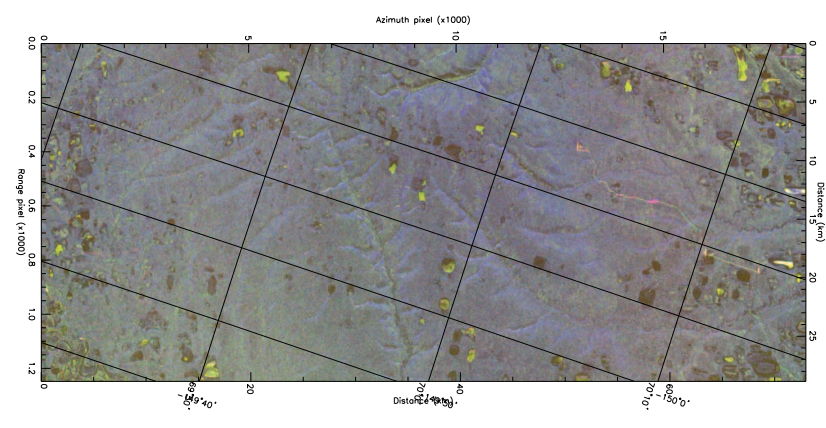

Fig. 1. Pauli image of the test site (red: dihedral, green: volume and blue: surface)

the satellite, so that $S$ stands for the distance to the satellite. Once we define TEC $\stackrel{\text { def }}{=} \int N \mathrm{~d} l$, we get

$$
\Omega=\zeta \frac{e \vec{B} \cdot \hat{\kappa}}{c m f_{0}^{2}} \mathrm{TEC} .
$$

As a consequence of Eq. (2), the polarization ellipse of the trans/received pulses are rotated by $\Omega$ as they propagate through the ionosphere. Accordingly the measured scattering matrix $\mathbf{S}$ is distorted. Each channel of a linearly polarized quad-pol SAR is intermixed under the FR of $\Omega$ as follows

$$
\begin{aligned}
S_{h h}(\Omega) & =\cos ^{2} \Omega S_{h h}-\sin ^{2} \Omega S_{v v}, \\
S_{h v}(\Omega) & =S_{x x}+\cos \Omega \sin \Omega\left(S_{h h}+S_{v v}\right), \\
S_{v h}(\Omega) & =S_{x x}-\sin \Omega \cos \Omega\left(S_{h h}+S_{v v}\right), \\
\text { and } S_{v v}(\Omega) & =-\sin ^{2} S_{h h}+\cos ^{2} \Omega S_{v v},
\end{aligned}
$$

where $S_{p q}(\Omega)$ and $S_{p q}$ represent the elements of the measured and the original scattering matrix, respectively, for transmitting polarisation $q$ and receiving polarisation $p$. Once $\Omega$ is estimated, the elements of the original scattering matrix $\mathbf{S}$ can be acquired from the measured scattering matrix $\mathbf{S}(\Omega)$ using Eqs. (6). The FR estimators are collected in [6].

FR can be estimated in the master and slave SAR data separately. Their difference is the differential FR, which can be converted to the differential TEC according to Eq. (5).

\subsection{Azimuth shift}

Azimuth variations of TEC induce azimuth shifts of focusing positions on SAR imagery. The position of azimuth focusing is determined by the time-Doppler relation. The ideal timeDoppler relation in the absence of ionospheric effects is [7]

$$
\frac{\Delta f}{f_{0}}=2 \frac{v_{\text {rel }}}{c}=\frac{2 v^{2} t}{R_{0} c}=\frac{D_{f}}{f_{0}} t
$$

where $\Delta f$ is Doppler frequency, $f_{0}$ is carrier frequency, $v_{\text {rel }}$ is the relative velocity between the antenna and the target, $v$ is the velocity of the SAR sensor, $R_{0}$ is the zero-Doppler distance, and $D_{f}$ is used for the Doppler rate.
When the TEC varies along the azimuth direction, an additional phase component is introduced into Eq. (7). It reads

$$
\frac{\Delta f}{f_{0}}=\frac{2 \pi \Delta \phi}{f_{0}}=-2 \zeta \frac{v_{\text {piercing }}}{c f_{0}^{2}} \frac{\partial \mathrm{TEC}}{\partial x},
$$

where $v_{\text {piercing }}$ is the speed of the piercing point. Because we are interested in the phase history of a single target, the relation $v: v_{\text {piercing }}=h_{\mathrm{S}}: h_{\text {iono }}$ holds. The disturbed Doppler history is obtained by adding Eq. (8) to Eq. (7). Assuming a zero-squint geometry, a target is focused at the azimuth time when the Doppler history becomes zero. The azimuth shift is found by

$$
\Delta a=\frac{2 \zeta v_{\text {piercing }} \text { PRF }}{c f_{0} D_{f}} \frac{\partial \mathrm{TEC}}{\partial x}
$$

in unit of azimuth pixel. While in the case of a single SAR acquisition azimuthal shifts are of secondary importace, in the case of InSAR they become critical. If the azimuth change rate of the ionosphere is different in the master and slave acquisitions, the focusing positions are no longer identical, leading to a coregistration offset, that may induce an additional decorrelation contribution in the interferogram.

\subsection{Interferometric phase}

The phase advances in the ionosphere disturb interferometric differential phases causing ionospheric phase screen, if the TEC changes between two acquisitions. The interferometric phase is unavoidable in the repeat-pass interferometry modes. Integrating Eq. (1) gives the interferometric phase $\phi$

$$
\phi=4 \pi \frac{\zeta \Delta \mathrm{TEC}}{c f_{0}} \text {. }
$$

\section{CORRECTIONS AND METRICS}

For our investigations we used interferometric ALOS PALSAR data acquired over the northern part of Alaska in spring 2007 (Apr. $1^{\text {st }}$ and May 17 $7^{\text {th }}$ ). The corresponding vertical wavenumber is $k_{z}=0.014 \mathrm{rad} / \mathrm{m}^{-1}$. The Pauli image of the master acquisition is shown in Fig. 1

\subsection{Faraday rotation}

Using Eqs. (6) the effect of FR can be corrected on each channel of quad-pol SAR data. For this we have used Bickel \& Bates estimator (Fig. 2). The correction efficiency can be tested by means of the reflection symmetry assumption.

The direct result of the reflection symmetry is the full decorrelation between co- and cross-pol channel, i.e. $\gamma_{h h-v h}=$ $\gamma_{v v-h v}=0$. Under FR the numerator of this coherence is

$$
\begin{aligned}
& \left\langle S_{h h}(\Omega) S_{v h}(\Omega)^{*}\right\rangle= \\
& \sin 2 \Omega\left(-\left\langle S_{h h} S_{h h}^{*}\right\rangle-2 i \Im\left\{\left\langle S_{h h} S_{v v}^{*}\right\rangle\right\}+\left\langle S_{v v} S_{v v}^{*}\right\rangle\right) / 4 \\
& -\sin 4 \Omega\left(\left\langle S_{h h} S_{h h}^{*}\right\rangle+2 \Re\left\{\left\langle S_{h h} S_{v v}^{*}\right\rangle\right\}+\left\langle S_{v v} S_{v v}^{*}\right\rangle\right) / 8 .
\end{aligned}
$$




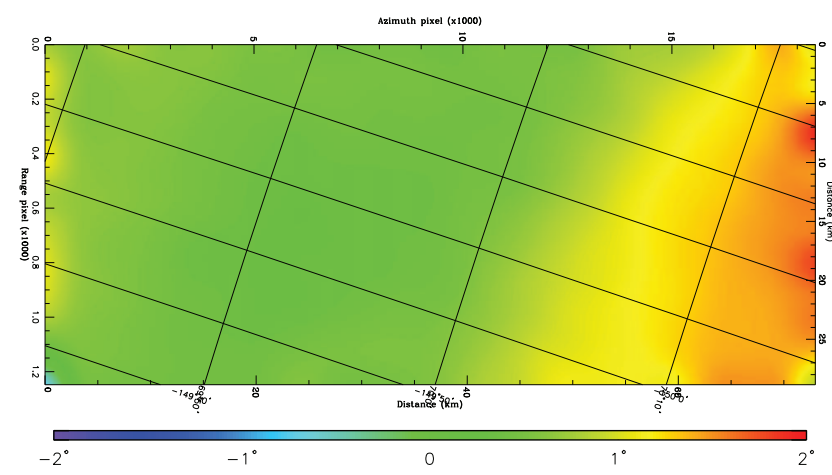

Fig. 2. Estimated differential Faraday rotation using Bickel \& Bates esitmator

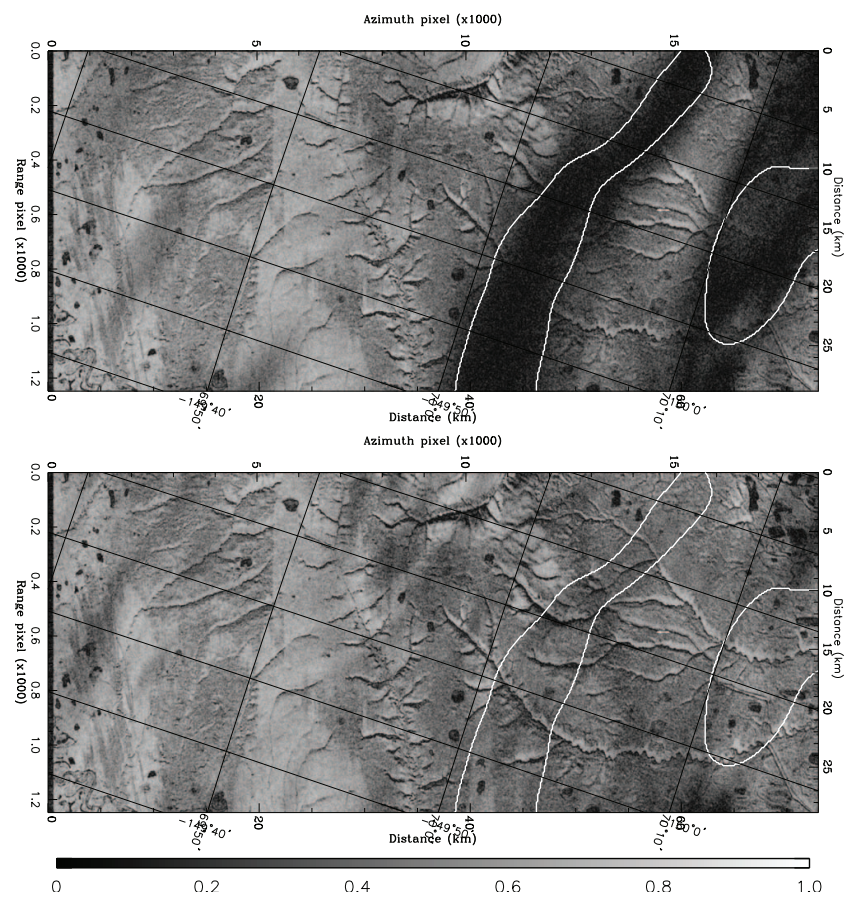

Fig. 3. Comparison of $\gamma$, before and after $\Delta a$ correction

The amplitude of the first term on the right hand side is small because of the opposite signs of comparable real numbers, i.e. $\left\langle S_{h h} S_{h h}^{*}\right\rangle$ and $\left\langle S_{v v} S_{v v}^{*}\right\rangle$. The amplitude of the dominant second term is a real number and it is proportional to $\sin 4 \Omega$. Except for azimuth slope effects, the co-/cross-pol coherence can provide a reliable FR correction performance metric.

\subsection{Azimuth shift}

The interferometric coherence is maximized when the two SAR images are exactly coregistrated. The interferometric coherence and the misregistration $\delta_{\mathrm{AZ}}$ are related by

$$
\gamma_{\mathrm{cor}}=\frac{\sin \pi \delta_{\mathrm{AZ}}}{\pi \delta_{\mathrm{AZ}}} .
$$
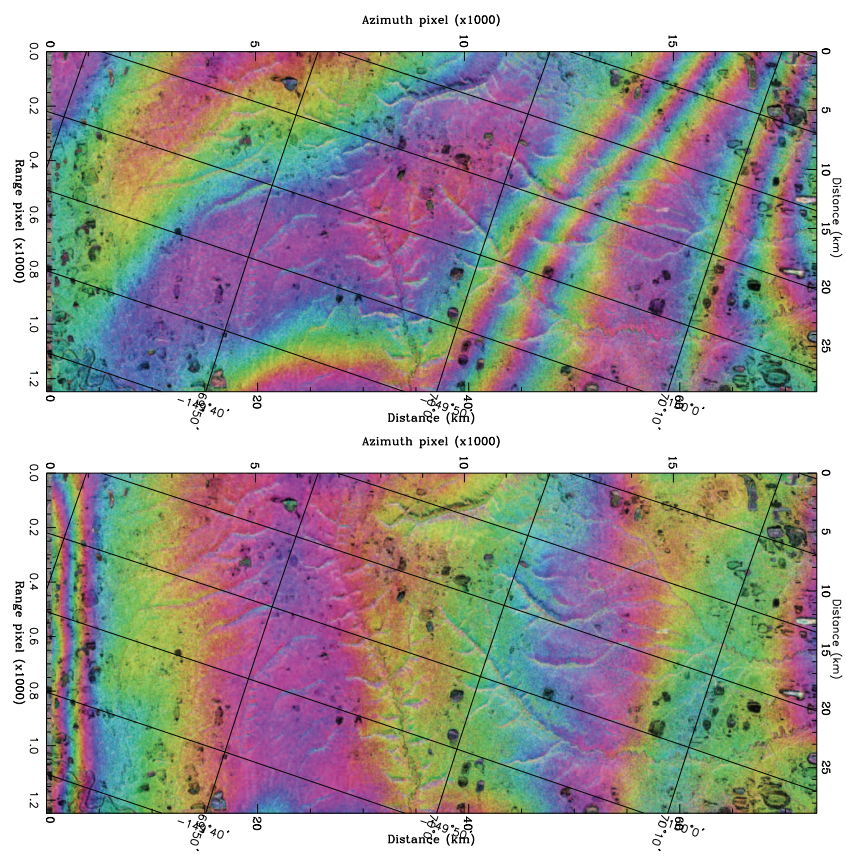

Fig. 4. Comparison of the interferometric phases, before and after correction using $\Delta \Omega$

The correlation of the master and slave images can provide the amount of misregistration, and this mismatch can be corrected by resampling the slave image. Fig. 3 shows the improvement of $\gamma$ before and after the slave resampling according to the estimated azimuth offsets induced by the (differential) ionosphere. The lost of $\gamma$ where TEC changes rapidly (in the white contours) is almost fully recovered.

\subsection{Interferometric phase}

The ionospheric phase screen can be compansated when accurate measurement of $\triangle \mathrm{TEC}$, which can be achieved from $\Omega$ using Eq. (5), are available. Fig. 4 shows the correction of the ionospheric phase using $\Delta \Omega$. A residual systematic phase term is visible even after correction.

\section{COMBINED TEC ESTIMATOR}

The estimation result from $\Delta \Omega$ provides the information about $\Delta$ TEC (Section 2.2), and the relative azimuth shift estimation $\Delta a$ is proportional to $\partial \Delta \mathrm{TEC} / \partial x$ (Section 2.3). The improvement of $\Delta \mathrm{TEC}$ estimation using their information is described in this section.

Let us assume that an interval $I=[-L / 2, L / 2]$ laid parallelly to the azimuth direction on the ionosphere, where $L$ is the coherence length at around the ionospheric altitude and the origin is placed in the zero-Doppler plane. The mean of $\triangle$ TEC in the interval $I$ determines the $\Delta \Omega$ and its linear change rate determines $\Delta a$. Dividing the interval $I$ into 


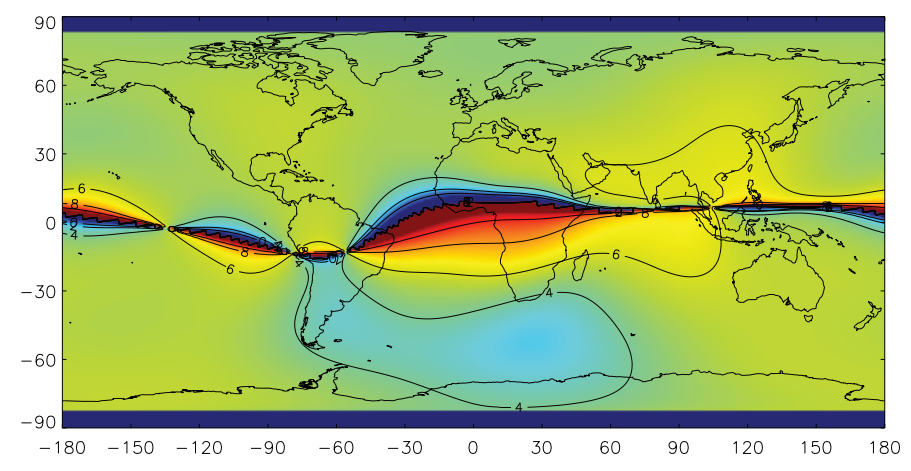

Fig. 6. Sensitivity of the interferometric phase depending on the altitude variation of the ionosphere at L-band. TEC is fixed at 1 TECU, and the altitude changes from 400 to 410 $\mathrm{km}$.

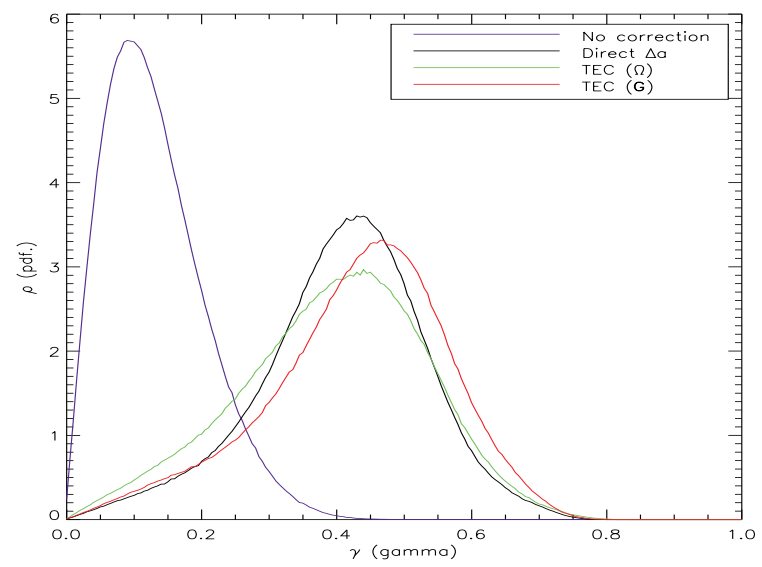

Fig. 5. Change of $\gamma$ before (violet) and after several $\Delta a$ corrections (black: direct $\Delta a$ estimation, green: $\triangle$ TEC from $\triangle \mathrm{FR}$, and red: $\triangle \mathrm{TEC}$ from combined estimator).

$K$ segments, we can allocate $\triangle \mathrm{TEC}$ for every segment in the interval. Then FR at the zero-Doppler plane is

$$
\Delta \Omega=\zeta \frac{e \vec{B} \cdot \hat{\kappa}}{c m f_{0}^{2}} \frac{1}{K}\left(\begin{array}{llll}
1 & 1 & \cdots & 1
\end{array}\right) \Delta \overrightarrow{\mathrm{TEC}} .
$$

At the same time, the change rate of $\triangle \mathrm{TEC}$ is

$$
\begin{aligned}
\frac{\partial \Delta \mathrm{TEC}}{\partial x} & =\frac{12}{L\left(K^{2}-1\right)}\left(\begin{array}{llll}
1 & 2 & \cdots & K
\end{array}\right) \Delta \overrightarrow{\mathrm{TEC}} \\
& +\frac{6}{L(K-1)}\left(\begin{array}{llll}
1 & 1 & \cdots & 1) \Delta \overrightarrow{\mathrm{TEC}}
\end{array}\right.
\end{aligned}
$$

where $\Delta \overrightarrow{\mathrm{TEC}}=\left(\begin{array}{llll}\Delta \mathrm{TEC}_{1} & \Delta \mathrm{TEC}_{2} & \cdots & \Delta \mathrm{TEC}_{K}\end{array}\right)^{T}$. Eq. (14) is derived from the least square estimation. Having the observables $\Delta \Omega$ and $\Delta a$, an operator $\mathbf{G}$, which relates $\triangle \mathrm{TEC}$ to the observables, can be defined.

$$
\vec{d}_{\mathrm{obs}}=\left(\begin{array}{c|c}
\Delta \vec{\Omega} \\
\Delta \vec{a}
\end{array}\right)=\left(\begin{array}{c|c}
\mathbf{G}_{\Omega} & \mathbf{0} \\
\hline \mathbf{0} & \mathbf{G}_{\Delta a}
\end{array}\right) \Delta \overrightarrow{\mathrm{TEC}}=\mathbf{G} \Delta \overrightarrow{\mathrm{TEC}} .
$$

This is a typical formulation of an inverse problem [8]. As the equation is linear, taking its inverse is enough to find $\triangle \mathrm{TEC}$. After [8, p.67], the $\triangle \mathrm{TEC}$ can be found by

$$
\Delta \overrightarrow{\mathrm{TEC}}=\left(\mathbf{G}^{T} \mathbf{C}_{\mathrm{D}}^{-1} \mathbf{G}\right)^{-1}\left(\mathbf{G}^{T} \mathbf{C}_{\mathrm{D}}^{-1} \vec{d}_{\mathrm{obs}}\right),
$$

where $\mathbf{C}_{\mathrm{D}}$ stands for the covariance matrix of the observed data.

In order to define the performance of the combined estimator, we compared $\gamma \mathrm{s}$ after the azimuth resamplings according to i) direct $\Delta a$ estimation, ii) $\triangle \mathrm{TEC}$ estimation using $\Delta \Omega$, and iii) combined estimator. Fig. 5 shows the results. The combined estimator leads to the highest $\gamma$ followed by direct $\Delta a$ estimation and the $\Delta \mathrm{TEC}$ using $\Delta \Omega$.

\section{DISCUSSION \& CONCLUSION}

The above discussion was based on the assumption that the effective altitude of the ionosphere is constant with respect to time. In that case the discrepancies among TEC, $\partial \mathrm{TEC} / \partial x$ and $\phi$ are the result of random noise. However, as seen in Fig. 4, there is as systematic bias. A reason for this can be the variation of the ionospheric altitude with time.

Fig. 6 shows the sensitivity of the interferometric phase depending on the ionospheric altitude change. The numbers along contours indicate the interferometric phase in degrees for a constant TEC (1 TECU), and an ionospheric altitude that changes about $10 \mathrm{~km}$. We can see that in the case of interferometric phase corrections, we need the information about the vertical structure of the ionosphere in addition to the TEC.

\section{REFERENCES}

[1] D. P. Belcher, "Theoretical limits on SAR imposed by the ionosphere," IET Radar Sonar Navig., vol. 2, no. 6, 2008.

[2] Zheng-Wen Xu, Jian Wu, and Zhen-Sen Wu, "A survey of ionospheric effects on space-based radar," Waves in Random Media, vol. 14, pp. S189-S273, 2004.

[3] Franz Meyer, "Performance requirements for correction of ionospheric signals in L-band SAR data," in Proc. EUSAR 2010 8th European Conference on Synthetic Aperture Radar, Aachen, Germany, June7-10 2010.

[4] K. C. Yeh and C. H. Liu, Theory of Ionospheric Waves, Academic Press, 1972.

[5] Sir Edward Victor Appleton, "Wireless studies of the ionosphere," J. Inst. Electr. Eng., vol. 71, pp. 642-650, 1932.

[6] Jun Su Kim and Konstantinos P. Papathanassiou, "Faraday rotation estimation performance analysis," in proc. EUSAR2010 8th European Conference on Synthetic Aperture Radar, Aachen, Germany, June7-10 2010.

[7] John C. Curlander and Robert N. McDonough, RADAR Systems and Signal Processing, Wiley \& Sons, Inc., 1991.

[8] Albert Tarantola, Inverse Problem Theory, SIAM, 2005. 